EPJ Web of Conferences 106, 04012 (2016)

DOI: $10.1051 /$ epjconf/201610604012

(C) Owned by the authors, published by EDP Sciences, 2016

\title{
Estimation of Covariances on Prompt Fission Neutron Spectra and Impact of the PFNS Model on the Vessel Fluence
}

\author{
Léonie Berge ${ }^{\mathrm{a}}$, Olivier Litaize, Olivier Serot, Pascal Archier, Cyrille De Saint Jean, Yannick Pénéliau, \\ and David Regnier
}

CEA, DEN, DER, SPRC, Cadarache, 13108 Saint-Paul-lez-Durance, France

\begin{abstract}
As the need for precise handling of nuclear data covariances grows ever stronger, no information about covariances of prompt fission neutron spectra (PFNS) are available in the evaluated library JEFF-3.2, although present in ENDF/B-VII.1 and JENDL-4.0 libraries for the main fissile isotopes. The aim of this work is to provide an estimation of covariance matrices related to PFNS, in the frame of some commonly used models for the evaluated files, such as the Maxwellian spectrum, the Watt spectrum, or the Madland-Nix spectrum. The evaluation of PFNS through these models involves an adjustment of model parameters to available experimental data, and the calculation of the spectrum variance-covariance matrix arising from experimental uncertainties. We present the results for thermal neutron induced fission of ${ }^{235} \mathrm{U}$. The systematic experimental uncertainties are propagated via the marginalization technique available in the CONRAD code. They are of great influence on the final covariance matrix, and therefore, on the spectrum uncertainty band width. In addition to this covariance estimation work, we have also investigated the importance on a reactor calculation of the fission spectrum model choice. A study of the vessel fluence depending on the PFNS model is presented. This is done through the propagation of neutrons emitted from a fission source in a simplified PWR using the TRIPOLI- $4{ }^{\circledR}$ code. This last study includes thermal fission spectra from the FIFRELIN Monte-Carlo code dedicated to the simulation of prompt particles emission during fission.
\end{abstract}

\section{Introduction}

For reactor calculations, the expectations on nuclear data are becoming more and more important. Not only must they provide an acceptable reproduction of experimental observables, but the evaluators must also handle their covariances in a rigorous way. In the case of Monte-Carlo codes for neutronics, the main source of uncertainty comes from nuclear data. This is why an accurate estimation of the nuclear data covariance matrix is mandatory in order to master the covariances on neutronic parameters. Our study fits into this context, and focuses on the particular case of the Prompt Fission Neutron Spectra (PFNS), for which no covariances are given in the latest JEFF evaluation. We will present an estimation of the covariance matrix, applicable to any fissionning system, and to three PFNS models. However, the results will only be shown for thermal-neutron induced fission of ${ }^{235} \mathrm{U}$ in the frame of the

\footnotetext{
${ }^{\text {a }}$ Corresponding author: leonie.berge@cea.fr
}

This is an Open Access article distributed under the terms of the Creative Commons Attribution License 2.0, which permits unrestricted use, distribution, and reproduction in any medium, provided the original work is properly cited. 
Madland-Nix model [1]. These covariances will allow us in the near future to propagate the PFNS uncertainty on reactor calculation uncertainties. However, as a preliminary study we investigated the impact of the model, without any covariances involved, on reactor calculations. We will present a study of the neutron flux on a PWR vessel, depending on the ${ }^{235} \mathrm{U}$ thermal PFNS. This information is of major importance for the PWR reactor life-time prediction. In the sodium-cooled fast reactor domain, we also studied the influence of the PFNS on the penetration of fission neutrons through sodium. This study has been lead by the JANUS - Phase 8 experiment [2].

\section{PFNS Models Considered in the Present Study}

The PFNS is defined as the probability density function $\chi(E)$ for the prompt fission neutron kinetic energy, such that the neutron has a probability $\chi(E) \mathrm{d} E$ to be emitted with an energy lying between $E$ and $E+\mathrm{d} E$; it is therefore normalized to one. The simplest and most widely used model consists in approximating the PFNS with a Maxwellian function. It takes only one parameter, the "temperature" $T$, which is energy-homogeneous, and has the well-known form $\chi^{M}(E, T)=2 /\left(\sqrt{\pi T^{3}}\right) \sqrt{E} e^{-E / T}$. This model, though the simplest, is still used for some isotopes in the latest JEFF evaluation. The Watt spectrum [3] has two parameters, $T_{W}$ and $E_{f}$. It corresponds to the transformation of a Maxwellian center-of-mass spectrum into the laboratory frame, considering an average fragment moving with an average kinetic energy per nucleon $E_{f}$. The Madland-Nix model [1] is built from the Weisskopf theory of fission fragment evaporation. The spectrum of each fragment, light or heavy, is described by two parameters: the fragment maximal temperature $T_{m}$, assumed to be the same for light and heavy fragments $\left(\right.$ i.e. $T_{m}^{L}=T_{m}^{H}=T_{m}$ ), and the fragment kinetic energy per nucleon in the lab frame, $E_{f}^{L, H}$. The contributions of both fragments must be summed, and weighted by the ratio of the average fragment neutron multiplicity $\bar{v}_{L, H}$ over the total average prompt neutron multiplicity $\bar{v}_{L}+\bar{v}_{H}$. However, as the Madland-Nix spectrum is quite insensitive to this ratio, we set the contribution of the fragments to 50\% each. We finally consider the PFNS computed by the FIFRELIN code $[4,5]$, which simulates the whole process of fission fragment de-excitation. The code, developed at CEA-Cadarache, allows calculating several prompt fission particle-related properties, in the frame of one consistent probabilistic model. This approach relies on a more refined model for energy repartition between the fragments and for the fragment de-excitation process. Two de-excitation modes are implemented: the Weisskopf model for neutrons (FIFRELIN-W) and a Hauser-Feshbach model for neutrons and gammas (FIFRELIN-HF).

To visualize the PFNS from different models, Figure 1 shows the difference between several PFNS and the ENDF/B-V evaluated PFNS for ${ }^{235} \mathrm{U}$ thermal fission. This last PFNS has been chosen as reference because it is widely used in nuclear industry for shielding calculations involving a fission source; it corresponds to a Watt spectrum $\left(T_{W}=0.99 \mathrm{MeV}, E_{f}=0.55 \mathrm{MeV}\right)$. Among the spectra shown on Fig. 1, we show a Maxwellian spectrum $(T=1.32 \mathrm{MeV})$; the Watt spectrum resulting from work presented in Sect. $3\left(T_{W}=1.01, E_{f}=0.48 \mathrm{MeV}\right)$; the Watt spectrum from Cranberg $\left(T_{W}=0.97 \mathrm{MeV}\right.$, $E_{f}=0.53 \mathrm{MeV}$ ) which is also commonly used for reactor calculations; the Madland-Nix spectrum resulting from work presented in paragraph $3\left(T_{m}=1.01 \mathrm{MeV}, E_{f}^{L}=0.93 \mathrm{MeV}, E_{f}^{H}=0.29 \mathrm{MeV}\right)$. The JEFF-3.2 PFNS for ${ }^{235} \mathrm{U}\left(\mathrm{n}_{\mathrm{th}}, \mathrm{f}\right)$ was also included, it is based on the Madland-Nix model, and is similar to the ENDF/B-VII.1 and JENDL-4.0 PFNS (as can be seen later on Figure 2a). Finally, we included the thermal ${ }^{235} \mathrm{U}$ PFNS computed by FIFRELIN-W. The average prompt fission neutron kinetic energy, computed with each of these models, is also shown in Fig. 1.

\section{Generation of the PFNS Variance-covariance Matrix Using CONRAD}

CONRAD [6] is a code developed at CEA-Cadarache, devoted to nuclear data evaluation. In particular, it allows adjusting the free parameters of theoretical models to measured data through the 


\section{$15^{\text {th }}$ ISRD}

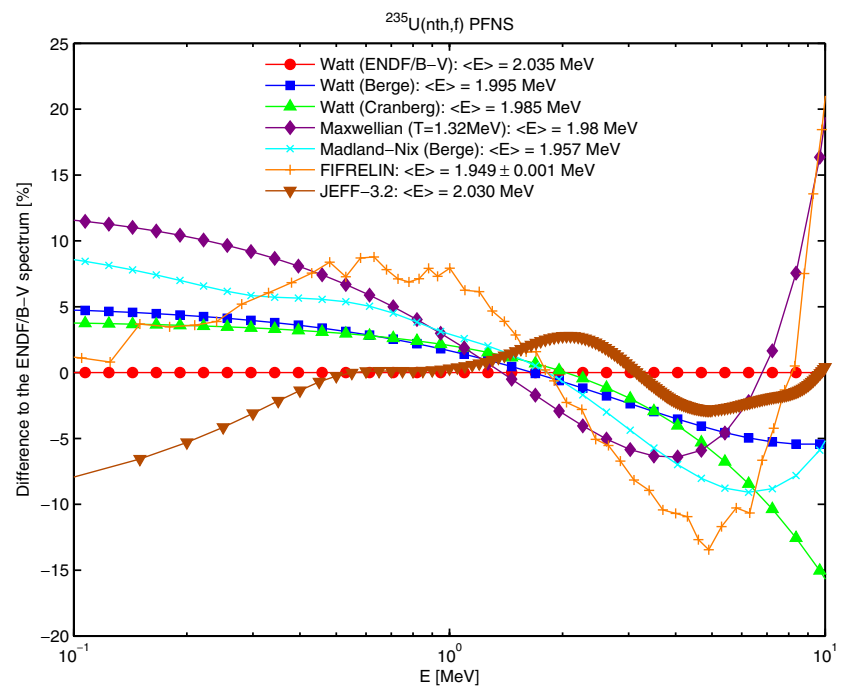

Figure 1. Relative difference between several PFNS and the ENDF/B-V PFNS. The legend indicates the average prompt fission neutron kinetic energy.

Bayesian technique. The three models (Maxwellian, Watt and Madland-Nix) mentioned above were implemented and the model parameters were adjusted to the available experimental data with their random counting uncertainty. The FIFRELIN model of PFNS has no analytical form since it comes from a Monte-Carlo simulation, therefore it was not part of the present study. For Watt and MadlandNix models, the prior parameter values were computed as suggested by Madland and Nix in reference [1]. The Maxwellian parameter $T$ prior value was set as commonly suggested in the literature namely, $1.32 \mathrm{MeV}$ for ${ }^{235} \mathrm{U}$ thermal fission, $1.38 \mathrm{MeV}$ for ${ }^{239} \mathrm{Pu}$ thermal fission, or $1.42 \mathrm{MeV}$ for ${ }^{252} \mathrm{Cf}$ spontaneous fission. The prior uncertainty on the model parameters were put at $10 \%$ for $T, T_{W}$ and $T_{m}$, and $7 \%$ for $E_{f}, E_{f}^{L}$ and $E_{f}^{H}$, as suggested in reference [7]. No prior correlation was considered between the model parameters. After the adjustment, we obtain the best-fit values $\vec{x}_{\text {adj. }}$ for the model parameters, and their covariance matrix $M_{x}^{\text {adj. }}$. However, there is a second set of parameters that influence the adjustment, called the "nuisance" parameters $\vec{\theta}$. They are related to experimental spectra used in the adjustment; a few examples are normalization, background, or detection efficiency. Their covariances need to be propagated to our calculated spectrum, although we want the calculated spectrum to depend only on model parameters $\vec{x}$. After the adjustment, we use the marginalization technique [8] available in CONRAD to make the covariances of model parameters bear the covariances of nuisance parameters. If $G$ is the matrix containing the derivatives of the PFNS to all parameters (model parameters and nuisance parameters), and $M$ is the covariance matrix of all these parameters, then the PFNS covariance matrix is $M_{\mathrm{PFNS}}=G M G^{\mathrm{T}}$. The idea of the marginalization is to find a covariance matrix $M_{x}^{\text {marg. }}$ for model parameters which allows to reproduce the same covariance matrix $M_{\text {PFNS }}$, i.e. we want $M_{x}^{\text {marg. }}$ such that $G M G^{\mathrm{T}}=G_{x} M_{x}^{\text {marg. }} G_{x}^{\mathrm{T}}$. This gives us [9]:

$$
M_{x}^{\text {marg. }}=M_{x}^{\text {adj. }}+\left(G_{x}^{\mathrm{T}} \cdot G_{x}\right)^{-1} \cdot G_{x}^{\mathrm{T}} \cdot G_{\theta} \cdot M_{\theta} \cdot G_{\theta}^{\mathrm{T}} \cdot G_{x} \cdot\left(G_{x}^{\mathrm{T}} \cdot G_{x}\right)^{-1}
$$

$M_{\theta}$ is the covariance matrix of the marginalized parameters (normalization, background, etc.), and $G_{\theta}$ is the matrix containing the derivatives of the spectrum to the marginalized parameters. When using the marginalization, the experimental covariance matrix used in the adjustment - before marginalization must not account for the covariances of marginalized parameters, in order not to double-count them. It is important to note that after marginalization, the final PFNS has no dependence to the nuisance 


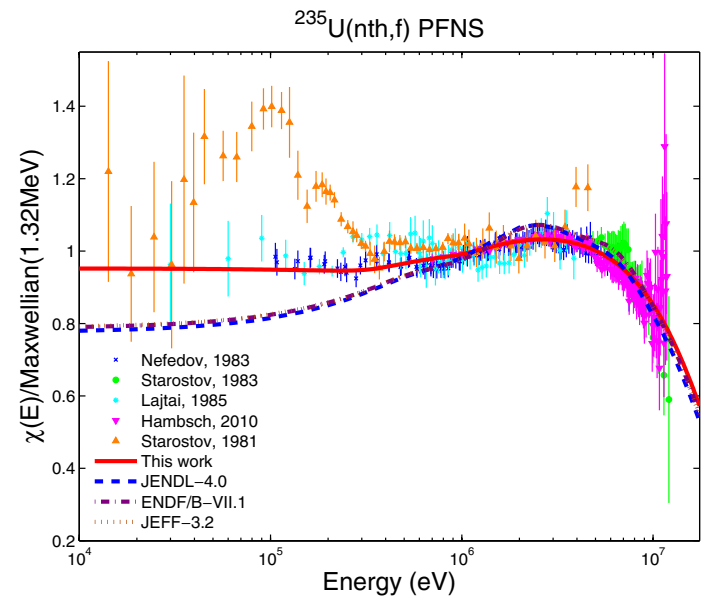

(a)

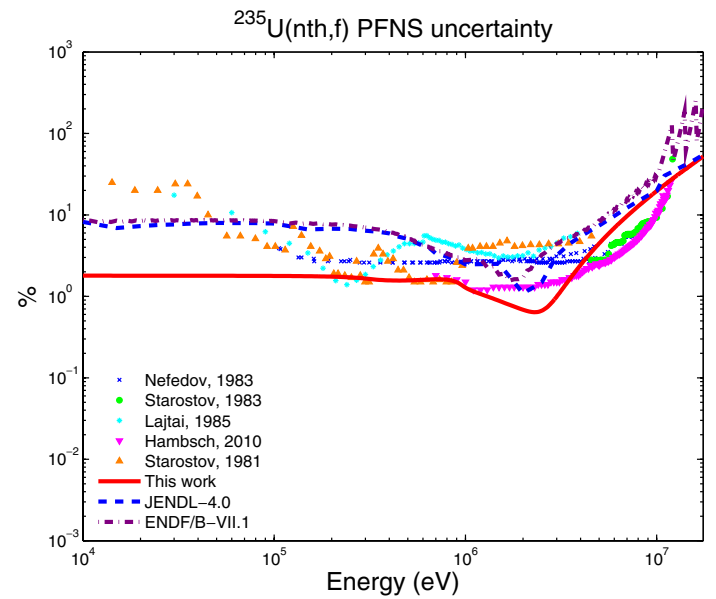

(b)
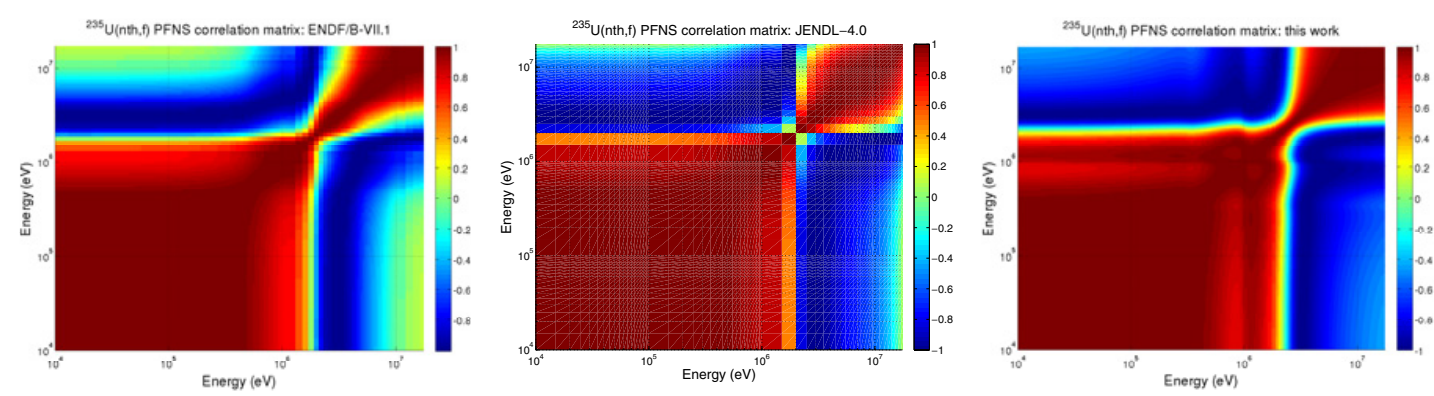

(c)

Figure 2. Results of the calculation for the Madland-Nix PFNS of ${ }^{235} \mathrm{U}$ (thermal neutron induced fission), marginalizing the experimental normalization (see text for full details). The PFNS obtained is compared to various libraries (a). The uncertainties (b) and correlations (c) resulting from this work are compared to those from JENDL4.0 and ENDF/B-VII.1.

parameters, and only depends on the model parameters. The PFNS remains a pure Maxwellian, Watt or Madland-Nix PFNS; only the covariance matrix of the model parameters is influenced by the marginalization. Even though the marginalization could be used for representing model defects, in this application it is used to account for experimental systematic uncertainties.

We will present here the results for thermal neutron-induced fission of ${ }^{235} \mathrm{U}$. The experimental spectra that we used to calculate the covariance matrix of ${ }^{235} \mathrm{U}$ thermal fission were those from Nefedov et al., 1983 (EXFOR entries 40871007 and 40871008), Starostov et al., 1981 (ref. [10]), Starostov et al., 1983 (EXFOR entry 40872004), Lajtai et al., 1985 (EXFOR entry 30704003) and Hambsch et al., 2010 [11]. We marginalized the experimental normalization of the measurements. The calculated PFNS has a norm constrained to one by construction; marginalizing on the experimental normalization may not seem consistent. However, experimental spectra used for the adjustment are constructed most of the time with several experiments not covering the whole energy range and as a result with potential normalization issues. For this reason, marginalizing the normalization, by adding an additional degree of freedom, could be seen as a first attempt to allow the experimental spectra to be consistent one with another. The uncertainty on the normalization of each experimental spectrum was estimated, based on the statistics collected in the experiment of interest; no correlation was considered between the different experiments, i.e. $M_{\theta}$ is diagonal. The resulting PFNS value 


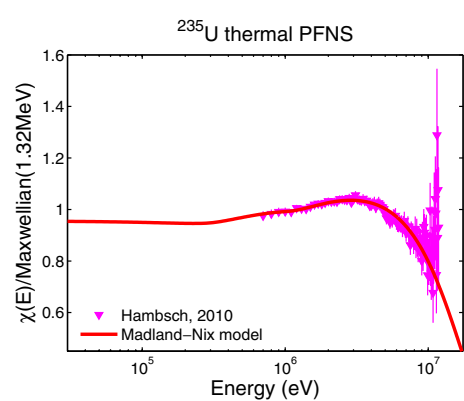

(a)

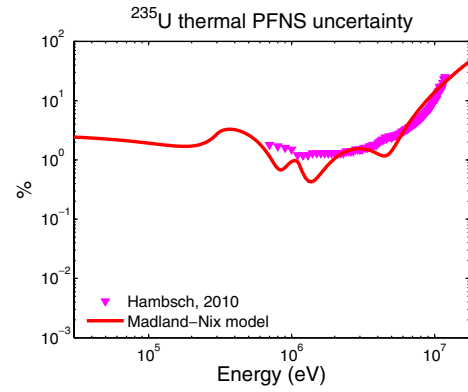

(b)

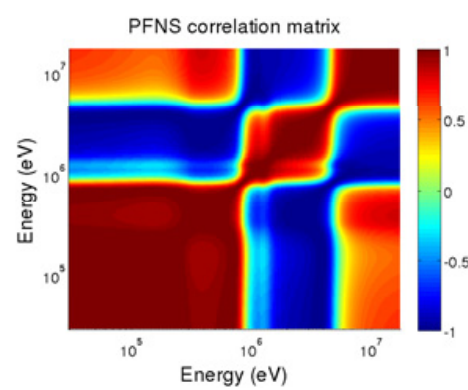

(c)

Figure 3. Madland-Nix PFNS of ${ }^{235} \mathrm{U}$ (thermal neutron induced fission), marginalizing the experimental detection efficiency, calculated with the data from Hambsch [11] (see text for full details).

relative to a Maxwellian $(T=1.32 \mathrm{MeV})$, standard deviation $\left(\sigma_{i}=\sqrt{\operatorname{cov}(i, i)}\right)$ and correlation matrix $\left(\operatorname{corr}(i, j)=\operatorname{cov}(i, j) /\left(\sigma_{i} \cdot \sigma_{j}\right)\right)$ are shown in Fig. 2. The experimental uncertainties represented are the ones given by the experimentalists. To save space, we will only present the results using the MadlandNix model, for thermal fission of ${ }^{235} \mathrm{U}$. The PFNS standard deviation as a function of the neutron energy is of the order of the experimental uncertainties. The PFNS shows high correlation for energies on the same side of about $2 \mathrm{MeV}$, and high anti-correlation for energies on different sides of this energy. This is the kind of pattern one can expect for a unity-normalized quantity. We can also see the PFNS taken from the three evaluations JEFF-3.2, ENDF/B-VII.1 and JENDL-4.0 (Fig. 2a), as well as uncertainties (Fig. 2b) and correlations (Fig. 2c) in the case of ENDF/B-VII.1 and JENDL-4.0. The three libraries give a PFNS based on the Madland-Nix model. The PFNS from this work is closer to the measurements at low energy (below $1 \mathrm{MeV}$ ). However, we can see from Fig. $2 \mathrm{~b}$ that the Madland-Nix model does not allow for the uncertainty to follow the shape of the experimental uncertainty, especially below $1 \mathrm{MeV}$.

It is also possible to marginalize the energy-dependent neutron detection efficiency, if we have this information from the experimentalists. Since the marginalization technique works on experimental parameters, it is necessary to approximate the experimental efficiency with a function of some parameters; these parameters will be marginalized. Figure 3 shows the preliminary results for the thermal neutron-induced fission of ${ }^{235} \mathrm{U}$ calculated with the experimental data from Hambsch [11] (experimental PFNS and detection efficiency). The shape of the calculated PFNS uncertainty and correlation as a function of neutron energy is changed compared to Fig. $2 b$, due to the energy dependence of the efficiency. To obtain a realistic result one must take into account as many measurements as possible. This preliminary result using only one PFNS shows the influence of the experimental parameters and their uncertainty.

\section{Preliminary Study of the Sensitivity to the PFNS of a PWR Vessel Fluence}

Producing more accurate nuclear data models and more complete covariance matrices is not useful if the neutronic observables we are interested in are not sensitive to them. Before propagating the PFNS covariances to reactor calculation uncertainties, we investigated the sensitivity of important neutronic parameters, like the vessel fluence, to the PFNS shape. The Monte-Carlo code TRIPOLI- $4{ }^{\circledR}[12]$ was used to compute the vessel flux, on the vessel inner side on a simplified PWR benchmark, with nuclear data mainly from JEFF-3.1.1. The geometry is a simplified quarter core, surrounded by water, the hot and cold water separated by a stainless steel envelope. The steel vessel envelopes the whole, and is 

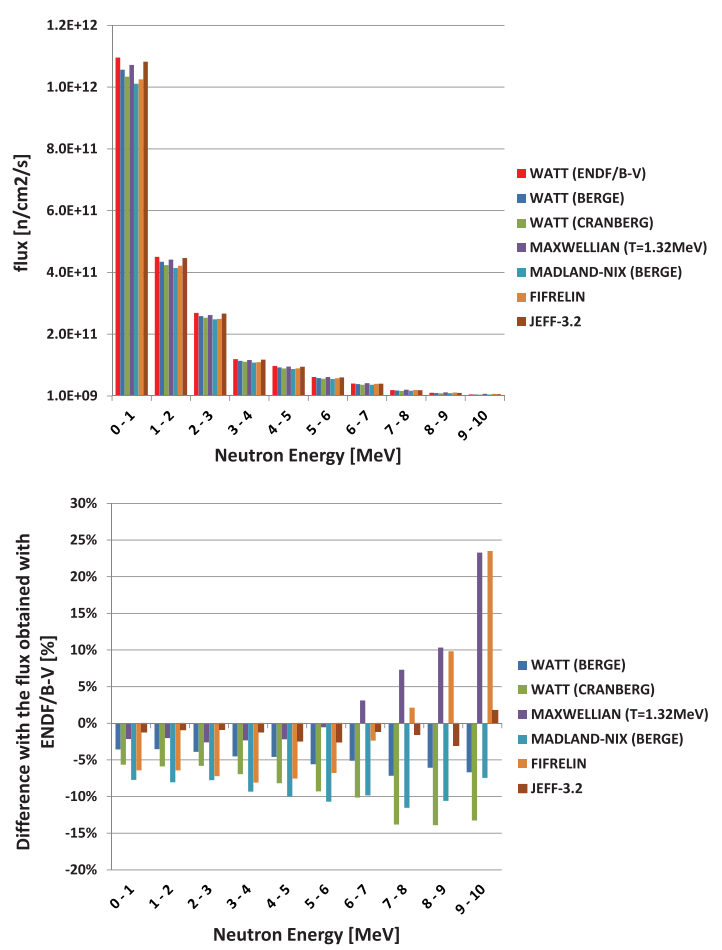

Figure 4. Neutron flux on the vessel, computed by TRIPOLI- $4^{\circledR}$. The graph on top shows the absolute flux for each PFNS, while the bottom graph shows the relative difference between the flux obtained with each PFNS and the flux obtained with the ENDF/B-V PFNS. The bars are in the same order than the legend, i.e. for each energy group, the left bar corresponds to the Watt spectrum from this work, the bar on its right corresponds to the Watt spectrum from ENDF/B-V, etc.

separated from the concrete layer by vacuum. The neutrons propagate from the core to the vessel. The calculation was performed several times, each time using a different energetic distribution of the neutron source, corresponding to different PFNS for thermal-induced ${ }^{235} \mathrm{U}$ fission. The PFNS that were tested on this benchmark are the ones presented in paragraph 2, and shown in Fig. 1. Figure 4 shows the resulting vessel flux, as well as the difference between the flux obtained for each PFNS and the flux obtained with the ENDF/B-V PFNS. We observe that the flux variation in each energy group, due to the change of the energetic distribution of the neutron source, is of the order of a few percent. The biggest discrepancies appear above $8 \mathrm{MeV}$ and are very large above $10 \mathrm{MeV}$; however, the vessel flux at these energies is very small. For all the spectra tested, the vessel flux below $6 \mathrm{MeV}$ is lower than in the case of the ENDF/B-V PFNS.

It would be interesting to also compute the sensitivity of the calculated displacement per atom (dpa) to the changes in PFNS. The difference in the flux is more important for high neutron energies; high energy neutrons are less frequent, but they cause more damage to the vessel. It is planned to compute the dpa associated to the different PFNS as well.

\section{Preliminary Study of the Prompt Fission Neutrons Propagation Through Sodium Depending on the PFNS}

The JANUS - Phase 8 experiment [2], performed in the NESTOR core in Winfrith, UK, was designed to measure the penetration of neutrons in sodium, as a support for the sodium-cooled fast neutron 


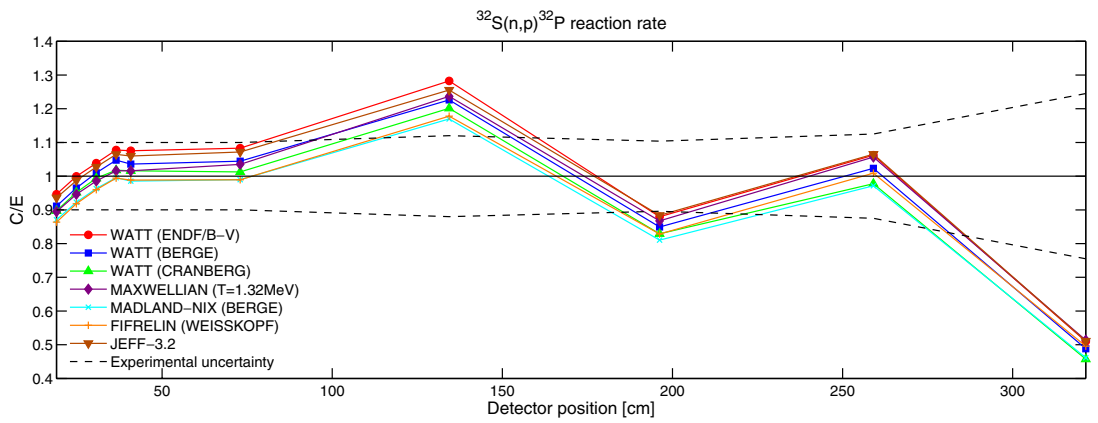

Figure 5. Calculated value over measured value $(C / E)$ for the $(n, p)$ reaction rate in the sulfur detector in the JANUS-8 experiment.

reactor development. The fission source was a ${ }^{235} \mathrm{U}$-enriched plate placed in the thermal neutron beam from NESTOR. The fission neutrons propagated through sodium tanks. Neutron detectors such as ${ }^{103} \mathrm{Rh}\left(\mathrm{n}, \mathrm{n}^{\prime}\right){ }^{103 \mathrm{~m}} \mathrm{Rh}$ or ${ }^{32} \mathrm{~S}(\mathrm{n}, \mathrm{p}){ }^{32} \mathrm{P}$ detectors, were placed at different distances from the fission source. The experiment has been modelled with TRIPOLI- $4{ }^{\circledR}$ [2]. The reaction rates in the detectors were computed for different energetic distributions of the fission source, corresponding to the same PFNS that were studied in paragraph 4 . We compared the calculated reaction rates to the measured reaction rates, as a function of the distance to the source. The results for the ${ }^{32} \mathrm{~S}$ detectors is shown in Fig. 5. The $C / E$ values show the same tendency for every PFNS as a function of the distance; however we see some influence of the PFNS. The ENDF/B-V PFNS leads to the highest reaction rates, especially for small distances - this PFNS has the highest mean neutron energy and highest component in the 2-6 MeV range. For low distances on the other hand, the Maxwellian and the FIFRELIN-W PFNS lead to the lowest reaction rates. For larger distances, the sulfur detector being sensitive to fast neutrons, the situation is reversed (high component above $7 \mathrm{MeV}$ for these two PFNS, see Fig. 1).

\section{Conclusion and Perspectives}

The CONRAD code allows in a single calculation the adjustment of nuclear data model parameters, and the generation of the covariance matrix in the frame of this model. We presented the PFNS covariance matrix obtained for thermal fission of ${ }^{235} \mathrm{U}$. The systematic uncertainty is propagated via the marginalization technique. The uncertainty of the final spectrum is of the same order than the uncertainties on the PFNS measurements used for the study, although the shape of the uncertainty as a function of neutron energy strongly depends on the PFNS model under study. The systematic uncertainty included in our calculation comes from the experimental uncertainty on normalization; we also show the influence of the neutron detection efficiency uncertainty. It is intended in the future to generate the covariance matrix associated to the PFNS from the FIFRELIN probabilistic code. To improve the PFNS accuracy and predictive power, CONRAD will be used to optimize the model parameters involved in the Weisskopf model in FIFRELIN, in order to account for the energy-dependence of the inverse crosssection in particular.

As an illustration for the need to generate the PFNS covariances, we showed a Monte-Carlo calculation of the neutron flux received by a PWR vessel, depending on the PFNS used for thermal fission of ${ }^{235} \mathrm{U}$. A study of the contribution of source neutrons, depending on their energy at their creation, on the vessel neutron flux, is underway. The sensitivity of the vessel flux to a change of PFNS, or to a change of PFNS model parameters, would then be deduced directly, without having to run any additional simulations. This has been calculated since the writing of the present paper. We also showed the impact of the PFNS on fission neutron penetration through sodium, using the JANUS - Phase 
8 experiment. The discrepancy between calculated and measured reaction rates in the detector shows the same rough trends as a function of penetration for every PFNS. No clear improvement of the $C / E$ values arises from the study, although the sensitivity to the PFNS is non negligible. Again, it is planned to use in the near future the Green functions in TRIPOLI- $4^{\circledR}$ to compute the contribution of the neutron birth energy to the reaction rate in the detector. It will be possible to give the change in PWR vessel flux, and in neutron penetration through sodium, associated to the PFNS recently available from IAEA [13].

We wish to thank F.-J. Hambsch and N. Kornilov for providing us experimental data on ${ }^{235} \mathrm{U}\left(\mathrm{n}_{\mathrm{th}}, \mathrm{f}\right)$.

\section{References}

[1] D.G. Madland, J.R. Nix, Nucl. Sci. Eng. 81, 213 (1982)

[2] P. Archier et al., JEF/DOC-1247 (2008); A. Avery, report NEA 1517/42 (1998)

[3] B. Watt, Phys. Rev. 87, 1037 (1952)

[4] O. Litaize, O. Serot, Phys. Rev. C 82, 054616 (2010)

[5] D. Regnier, O. Litaize, O. Serot, Physics Procedia 47, 47 (2013)

[6] P. Archier et al., Proc. of the Int. Conf. on Nucl. Data for Sc. and Tech. - ND2013 (2013)

[7] P.J. Griffin, J.G. Williams, Tech. Rep. SAND98-3662C (2000)

[8] B. Habert, C. De Saint Jean, G. Noguére, Nucl. Sci. Eng. 166, 276 (2010)

[9] P. Archier, Ph.D. thesis, Université de Grenoble (2011)

[10] B. Starostov, A. Semenov, V. Nefedov, Rep. IAEA INDC(CCP)-164/L (1981)

[11] F.-J. Hambsch, N. Kornilov, private communication (2014)

[12] E. Brun et al., Tech. Rep. CEA-R-6316 (2013)

[13] https://www-nds.iaea.org/pfns/public.html 